特別講演

\title{
The Current Role of the Supracricoid Partial Laryngectomies in the Management of Endolaryngeal Cancer
}

\author{
Ollivier Laccourreye
}

In this one hour keynote lecture the speaker will review the historiscal aspects of the supracricoid partial laryngectomies namely the cricohyoidoepiglottopexy (CHEP) and cricohyodopexy (CHP) procedure together with the technical reffinements that allow for a succesfull functional outcome. The indications, contraindications and oncological results published in the medical litterature as well as the experience gained at the author institution over the past thirty years will be presented and discussed. Emphasis, using numerous videos, will be placed upon the selection criteria, technical perioperative maneuvers and postoperative management that allow for a successfull outcome in terms of swallowing, respiration and phonation.

（抄録） 